\title{
Ethical Considerations for Planetary Protection in Space Exploration: A Workshop
}

\author{
J.D. Rummel, ${ }^{1}$ M.S. Race, ${ }^{2}$ G. Horneck, ${ }^{3}$ and the Princeton Workshop Participants
}

\begin{abstract}
With the recognition of an increasing potential for discovery of extraterrestrial life, a diverse set of researchers have noted a need to examine the foundational ethical principles that should frame our collective space activities as we explore outer space. A COSPAR Workshop on Ethical Considerations for Planetary Protection in Space Exploration was convened at Princeton University on June 8-10, 2010, to examine whether planetary protection measures and practices should be extended to protect planetary environments within an ethical framework that goes beyond "science protection" per se. The workshop had been in development prior to a 2006 NRC report on preventing the forward contamination of Mars, although it responded directly to one of the recommendations of that report and to several peer-reviewed papers as well. The workshop focused on the implications and responsibilities engendered when exploring outer space while avoiding harmful impacts on planetary bodies. Over 3 days, workshop participants developed a set of recommendations addressing the need for a revised policy framework to address "harmful contamination" beyond biological contamination, noting that it is important to maintain the current COSPAR planetary protection policy for scientific exploration and activities. The attendees agreed that there is need for further study of the ethical considerations used on Earth and the examination of management options and governmental mechanisms useful for establishing an environmental stewardship framework that incorporates both scientific input and enforcement. Scientists need to undertake public dialogue to communicate widely about these future policy deliberations and to ensure public involvement in decision making. A number of incremental steps have been taken since the workshop to implement some of these recommendations. Key Words: Planetary protection-Extraterrestrial life-Life in extreme environments-Environment-Habitability. Astrobiology 12, 1017-1023.
\end{abstract}

\section{Introduction and Background}

$\mathbf{H}$ ISTORICALLY, the rationale for COSPAR ${ }^{1}$ planetary protection policy has been to avoid contamination of planetary environments by biological contaminants or terrestrial microbes that could compromise current or future scientific investigations, particularly those searching for indigenous life.

As researchers recognized the increasing potential for discovery of extraterrestrial organisms, authors from diverse disciplines have suggested the need to examine what foundational ethical principles should frame our collective space

${ }^{1}$ COSPAR: The Committee on Space Research, an international committee of the International Council for Science, provides scientific advice and serves as a consultative body to the UN Committee on the Peaceful Uses of Outer Space (COPUOS). activities and relationships as we explore celestial bodies beyond Earth (e.g., Hargrove, 1986; Rolston, 1986; McKay, 1990, 2009; Lupisella and Logsdon, 1997; Randolph et al., 1997; Cockell and Horneck, 2004; NRC, 2006, pp 13-14; etc.).

The 2006 National Research Council (NRC) report on preventing the forward contamination of Mars (PREVCOM) raised similar concerns about possible ethical issues associated with the introduction of terrestrial organisms into sensitive extraterrestrial environments, even when current planetary protection policy is followed and suitable controls are used. Although acknowledging that ethical issues were beyond the scope of the committee's work, the NRC report recommended that ethical considerations be addressed in an international workshop to consider whether planetary protection polices for Mars should extend beyond protecting the science to include protecting the planet. The notion of an international workshop on ethical considerations was also

\footnotetext{
${ }^{1}$ East Carolina University, Greenville, North Carolina, USA.

${ }^{2}$ SETI Institute-CSC, Mountain View, California, USA.

${ }^{3}$ Institute of Aerospace Medicine, DLR, Köln, Germany.
} 
discussed and endorsed by NASA's Planetary Protection Subcommittee $^{2}$ (2007), ESA's Planetary Protection Working Group (2005), a COSPAR Planetary Protection Workshop in Montreal (January 2008), the COSPAR Planetary Protection Panel (PPP; July 2008), and other groups (e.g., IAA, IISL, COSPAR's PEX) ${ }^{3}$. The formal proposal to convene a workshop on ethical considerations and planetary protection, which urged consideration of whether biological planetary protection measures and other current practices intending to preserve planetary environments should be extended beyond science per se to include a broader ethical and practical framework, was approved by the COSPAR Bureau and Council in 2008. Specifically, the Workshop was expected to examine

- The ethical implications and responsibility to explore Mars in a manner that minimizes the harmful impacts of those activities on potential indigenous biospheres (whether suspected or known to be extant),

- Whether revision to current planetary protection policies is necessary to address these concerns, and

- How best to involve the public in such a dialogue about the ethical aspects of planetary exploration.

\section{Workshop Charter, Logistics, and Subgroup Deliberations}

The COSPAR Workshop on Ethical Considerations for Planetary Protection in Space Exploration was convened at Princeton University, Princeton, New Jersey, on June 8-10, 2010. The invited participants included 26 individuals selected for their combined expertise and experience in areas relevant to space science and exploration, ethics, law, policy, diplomacy, and communications.

The goals of the meeting, outlined in a Workshop Charter, were to determine

- Whether planetary protection measures and other practices should be extended to protect other aspects of planetary environments within an ethical and practical framework that goes beyond "science protection,"

- If so, what would be the bases for such policy and ethical framework? and

- What other implications and responsibilities are engendered when seeking to explore outer space in a manner that avoids harmful impacts on potential indigenous biospheres and other aspects of a planetary body?

The workshop began with a series of tutorial presentations to ensure that all participants were aware of important information prior to discussions and deliberations. Tutorial topics included information on planetary protection policy and implementation (see COSPAR, 2011), a review of the 2006 NRC study on preventing forward contamination on Mars, prospects for finding extraterrestrial life in the solar system, possible impacts in the long term of contamination and human activities in space, ethical considerations in space exploration and perspectives on the moral status and ethical

\footnotetext{
${ }^{2}$ The NASA Planetary Protection Subcommittee is the successor to the former NASA Planetary Protection Advisory Committee. Both have reported their advice through the NASA Advisory Council.

${ }^{3}$ IAA, International Academy of Astronautics; IISL, International Institute for Space Law; PEX, COSPAR Panel on Exploration.
}

principles related to the value of life and environments, the legal landscape associated with space exploration, possible approaches to governance and management that blend ethical and scientific perspectives (e.g., the UN Convention on Biological Diversity, planetary parks), and proposed stepping stones toward global space exploration that acknowledge multiple stakeholders.

Subsequently, all participants were assigned to one of two subgroups, determined in advance to ensure that each comprised similar expertise. In addition to being guided by the Workshop Charter, each subgroup was assigned a designated chairperson who was asked to lead his or her group through the following list of additional questions in whatever way the group deemed appropriate:

- In addition to the current COSPAR planetary protection policy's protection of biological/organic-constituent science opportunities, should we conduct solar system exploration to minimize or eliminate other possible negative effects on those bodies, for example, on

- Potential (but currently undetected) indigenous biospheres in the farther future ( $>500$ years)?

- Non-living aspects of a body that do not affect scientific study (e.g., historical, scenic, etc.)?

- Other aspects of preservation/stewardship that would make sense to extend into the rest of the solar system?

- Which ethical constructs support/require the additional protections? Which might argue against them?

- What revisions or additions to current COSPAR planetary protection policies would be necessary to address these additional considerations/protections?

- Would a convention on planetary protection/harmful contamination extending Article IX of the UN Outer Space Treaty (in the manner of the Convention on Biodiversity) be feasible/desirable as a way to provide for elaborations of expanded planetary protection policies?

- How should we best provide for public engagement with solar system exploration and involve the public in a dialogue about the ethical aspects of planetary protection?

- What revisions or additions to current planetary protection policy should be considered in support of such a broader engagement/dialogue, if any?

- Is now a good time to be working on this? If not, then when?

After several hours of subgroup discussions, all participants reconvened in a plenary session to present their respective approaches and discuss preliminary responses to the assigned questions.

Afterward, participants separated again into their assigned subgroups and completed drafting their working suggestions for later presentation in a final plenary session.

\section{Overview of Preliminary Subgroup Findings}

The section below provides a question-by-question overview of the issues examined and suggestions reached by the subgroups. This information provided the starting point for subsequent plenary deliberations and eventually led to development of the workshop consensus recommendations. More detailed information and summaries of the subgroup discussions are provided in the COSPAR workshop report (Rummel et al., 2012). 


\section{Questions 1 and 2}

In addition to the current COSPAR planetary protection poli$c y^{\prime}$ s protection of biological/organic-constituent science opportunities, should we conduct solar system exploration to minimize or eliminate other possible negative effects? Which ethical constructs support/require the additional protections? Which might argue against them?

Both subgroups responded in the affirmative to the question of whether we should conduct solar system exploration in ways that minimize or eliminate other possible negative effects on celestial bodies (beyond prevention of biological contamination). Each subgroup agreed that it was important to first start with baseline ethical approaches and constructs as they apply to living and non-living entities on Earth, and also to draw from considerations of stewardship and protection on Earth. Both groups agreed that considerably more expert discussion is needed on the detailed questions of how the ethical principles used on Earth would apply in the context of outer space and varied future scenarios/activities by different sectors (science vs. other activities, governmental and nongovernmental).

\section{Question 3}

What revisions or additions to current COSPAR planetary protection policies would be necessary to address these additional considerations/protections?

Each subgroup agreed that revisions or additions to planetary protection policy are necessary to address ethical concerns, but there should be no attempts to revise the Outer Space Treaty language itself by modifying either Article IX (planetary protection provisions) or Article VI (liability provisions). Proposed revisions to integrate ethical considerations can likely be accomplished through COSPAR and its long-standing review and advisory processes, similar to the way that other changes to planetary protection policy have been made over the decades. Interestingly, the two subgroups approached the question in distinctly different ways-one focusing primarily on modifications of COSPAR policy language related to harmful contamination and the other suggesting adoption of different policy measures for different target bodies in the solar system.

\section{Question 4}

Would a convention on planetary protection/harmful contamination extending Article IX of the UN Outer Space Treaty (in the manner of the Convention on Biodiversity) be feasible/desirable as a way to provide for elaborations of expanded planetary protection policies?

Obviously there are numerous ways to accomplish changes or elaborations to policy that would address concerns about planetary protection and harmful contamination. Both subgroups agreed that no single option should be endorsed at this time. Both subgroups felt strongly that any modifications to policy should be handled without changing the scope of the UN Outer Space Treaty (United Nations, 1967) and without involving changes in intergovernmental agreements. The groups suggested the need for more detailed examination of various policy frameworks that may provide model strategies or approaches among multiple international bodies and users (e.g., orbital debris agreements
(United Nations, 2010), international convention on biodiversity, habitat and environmental management approaches, stewardship strategies and use guidelines, cumulative impact assessments, preservation and protection requirements).

\section{Question 5}

What revisions or additions to current planetary protection policy should be considered in support of such a broader engagement/dialogue, if any?

Neither subgroup addressed the details of this question in their deliberations, but both acknowledged its importance. It became apparent in discussions that how to integrate ethical considerations into policy actually has two distinct focal areas: (1) current planetary protection policy aimed at avoiding biological contamination and interference with science exploration (target bodies and control measures) (De Vincenzi et al., 1983), and (2) addressing the policy gap in the realm of environmental management and contamination control for all activities and users of celestial bodies. There was a general agreement that planetary protection policy as currently implemented should remain separate and focused on science, target bodies, and contamination control measures. It became apparent that an entirely separate and parallel policy is likely to be needed in addressing environmental management of large areas and/or entire bodies.

\section{Question 6}

How should we best provide for public engagement in solar system exploration and involve the public in a dialogue about the ethical aspects of planetary protection?

The participants recognized the need to acknowledge different cultural and societal dimensions of exploration and research in space, and the responsibility of scientists and others to discuss potential impacts of their planned activities in space. Since both subgroups had similar views on the importance of communicating with the general public on ethical aspects of planetary exploration, they decided to develop communication recommendations together. Thus, two communications experts (one from each subgroup) were given the task of drafting a set of recommendations for plenary consideration that focused on both the ethics of making future policies as well as ways to maintain broad and sustainable public dialogue (see Recommendation 9 and accompanying text in the section on consensus recommendations, below).

\section{Question 7}

Is now a good time to be working on this? If not, then when?

Participants agreed that now is an appropriate time to discuss ethical concerns and policy revisions, recognizing both the potential discovery of extraterrestrial life and the great increase in exploration activities anticipated in coming years.

\section{Final Consensus Workshop Recommendations}

In the final plenary session, all participants reconvened to deliberate on suggestions from the two subgroups and develop final workshop recommendations that integrated ideas from tutorials, subgroup sessions, and plenary discussions. The plenary discussions explored alternative acceptable steps for possible policy revisions, identified issues needing further attention, and developed a set of consensus 
workshop recommendations, which were then voted upon by all participants.

\section{Recommendations on expansion of planetary protection policy}

All participants agreed that it would be important as an initial step to advocate an appropriately expanded framework for COSPAR planetary protection policy/policies to address other forms of "harmful contamination" that go beyond biological and organic constituent contamination. Moreover, it is important to maintain the existing, effective planetary protection policy while examining in parallel how to address ethical considerations related to life/non-life, environmental management, and multiple uses in space.

Thus, the participants voted to adopt the following recommendations regarding the expansion of planetary protection policy and to convey them to COSPAR for their further consideration and action:

Recommendation 1: An expanded overall framework for COSPAR planetary protection policy/policies is needed to address other forms of "harmful contamination" than currently addressed (i.e., biological and organic constituent contamination). Such policy framework should be developed within the scope of the UN Outer Space Treaty (Article IX on harmful contamination).

Recommendation 2: COSPAR should maintain the current policy on biological planetary protection virtually intact, under an expanded framework/umbrella for overall protection policy.

Recommendation 3: COSPAR should add a separate and parallel policy to provide guidance on requirements/best practices for protection of non-living/non-life-related aspects of outer space and celestial bodies.

The participants also discussed the time frame of concern for potential harmful contamination, noting that obliquity cycles and long-term changes in celestial environments may impact habitability (especially on Mars). The topic was deemed beyond the focus of the workshop yet needing further consideration. In light of continuing uncertainties about the existence of extraterrestrial life and the potential survivability of terrestrial microbes on solar system bodies, the participants agreed that the time frame of concern for planetary protection and harmful contamination should be considered in more detail. In the meantime,

Recommendation 4: COSPAR should consider that the appropriate protection of potential indigenous extraterrestrial life shall include avoiding the harmful contamination of any habitable environment-whether extant or foreseeable-within the maximum potential time of viability of any terrestrial organisms (including microbial spores) that may be introduced into that environment by human or robotic activity.

\section{Integrating ethical considerations into COSPAR} Planetary Protection Policy statements

To begin the process of integrating ethical considerations into an expanded COSPAR Planetary Protection Policy framework, the participants agreed to recommend the fol- lowing specific changes in COSPAR wording related to life and non-life, to potential extraterrestrial life, and to contamination and disturbance of celestial environments:

Recommendation 5: In the COSPAR policy preamble on planetary protection, add wording (italics) to acknowledge the values of life and non-living things, as follows:

- referring to COSPAR Resolutions 26.5 and 26.7 of $1964 \ldots$.

- notes with appreciation and interest the extensive work done by the Panel... and its successors... and the Panel on Planetary Protection and

- acknowledges that

- life, including extraterrestrial life, has special ethical status and deserves appropriate respect because it has both intrinsic and instrumental values, and

- non-living things, including extraterrestrial things, likewise have value and deserve respect appropriate to their instrumental, aesthetic, or other value to human or extraterrestrial life

- and accepts that for certain space mission/target body combinations undertaken for scientific, exploration, or other purposes by any entity, controls on contamination and restrictions on modifications of the natural state of solar system bodies shall be imposed in accordance with a specified range of requirements based on the policy statements associated with each specific policy.

Additionally, to address issues of concern about potential extraterrestrial life in the context of current policy on biological planetary protection concern, add wording (italics) to the policy statements as follows:

- The conduct of scientific investigations of possible extraterrestrial life-forms, precursors, and remnants must not be jeopardized. In addition, Earth must be protected from the potential hazard posed by extraterrestrial matter carried by a spacecraft returning from an interplanetary mission. Inherent in the conduct of scientific, exploration, and other activities - whether by robotic or human missions - is the need to consider and appropriately protect potential extraterrestrial life. Therefore, for certain space mission/target planet combinations, controls on contamination shall be imposed in accordance with issuances implementing this policy.

Finally, to address parallel concerns about contamination and environmental disturbance to non-living aspects of the Moon and other celestial bodies, the participants noted the need to add/modify wording to policy statements as follows (italics):

- Inherent in the conduct of scientific, exploration, and other activities - whether by robotic or human missions-is the need to consider and appropriately protect the Moon and other celestial bodies. Therefore, for certain space mission/target planet combinations, controls on contamination and environmental disturbance shall be imposed in accordance with issuances implementing this policy.

\section{Considering frameworks for developing parallel policy} on environmental management and protection

Participants acknowledged the variety of possible frameworks that could be adopted in the development of future 
parallel policy for environmental management and stewardship on the Moon and other celestial bodies. Recognizing that further examination and deliberation are warranted before recommending a preferred path, they agreed on the need for continued study of ways to manage and protect celestial environments from harmful contamination and disturbance.

Recommendation 6: To make progress toward developing and refining the parallel policy described above, there should be continued study over the next several years of various useful structures and frameworks that could incorporate scientific input on one end and enforcement on the other, considering such possible options as

- Environmental impact assessments for screening activities on celestial bodies;

- An intergovernmental mechanism for management of space exploration and use;

- Ensuring that COSPAR and other groups have input on management guidelines through a scientific committee providing advice to a convention or appropriate process/structure;

- Possible designation, establishment, and monitoring of planetary parks and areas for other uses, both protected and not protected; and

- Determine appropriate jurisdiction over planetary ecosynthesis or other modifications (e.g., atmospheric or physical disturbances) where science and other uses might be threatened or in conflict.

\section{Need for further analysis and discussion of ethical and other issues in connection with planetary protection}

During this time of reanalyzing planetary protection policy, the participants agreed that a conservative approach is warranted. It was noted that the Precautionary Principle calls for further investigations before interference that is likely to be harmful to Earth and other extraterrestrial bodies, including extraterrestrial life and the contamination and disturbance of celestial environments.

Recommendation 7: COSPAR should set up a group (or future workshop) to further explore the ethical values (e.g., intrinsic and instrumental) that apply to life, non-life, and environments as well as to the different classes of target objects in our solar system to provide guidance for balancing the different interests. Additional details on what this next workshop will entail should be developed afterward. During this period, when COSPAR is reanalyzing planetary protection policy, a conservative approach to decisions regarding space exploration and activities is warranted.

\section{Process for exploring/accomplishing desired changes in policy (for example, considering a convention on harmful contamination)}

During the plenary session, participants discussed a variety of management options that could be useful in accomplishing the needed changes in policy. A straw-man approach was proposed for initiating development of a workable framework for planetary protection policy, suggesting that it might be useful to work toward a convention on "harmful contamination" of outer space and its prevention, with the goal of defining, extending, and implementing international law based on an elaboration of Article IX of the UN Outer Space Treaty, as well as to include provisions for the use of space environments (Article III). Such a convention might be organized in a manner similar to the UN Convention on Biodiversity, which has a comprehensive structure that includes scientific input on one end and enforcement on the other.

Participants also briefly discussed a variety of other management options and policy approaches (e.g., environmental impact assessments and pre-screening of activities, designation and monitoring of special areas on target bodies, establishment of space stewardship and environmental regulations, development of intergovernmental mechanisms or bodies responsible for balancing future exploration and use). Since members of both subgroups felt that a variety of policy methods and processes should be analyzed in greater detail, participants agreed that it would be important to study the possible procedural steps and management models over the next several years and return later with specific recommendations regarding possible ways to accomplish the desired changes in policy. The plenary group felt it would be inadvisable to recommend a particular model (e.g., a convention) at this time.

Recommendation 8: COSPAR should elaborate on management guidelines in interaction with organizations such as IISL and others to establish a framework for environmental stewardship on celestial bodies for submittal to the UN COPUOS for UN General Assembly consideration. This should apply additionally to the accepted regulations for preventing harmful planetary contamination, which currently only consider biological and organic chemical contamination. This could include the establishment of an intergovernmental mechanism and/or body that provides management of space exploration and use.

\section{Communication, public engagement, and dialogue about the ethical aspects of space exploration and planetary protection}

As part of its recommendations on matters related to communications and public engagement, the participants noted the following points:

- COSPAR should encourage its members and associated states to initiate and sustain a broad-reaching public dialogue about the ethical aspects of space exploration and planetary protection and conduct public engagement and public consultation efforts at national and/or regional levels concerning ethics in space exploration.

- COSPAR policy regarding space exploration and the preservation of outer space environments should take into account and reflect the international trend toward sincere consultation with a broad range of publics about the ethical and policy issues associated with space exploration, as has been put into practice for consultation about developments in biotechnology, nanotechnology, neuroscience, and so on in Europe, the United States, and Canada.

- Toward addressing the challenges of assessing and incorporating public opinion in policy and planning, 
COSPAR should ask its Panels on Planetary Protection (PPP) and Exploration (PEX) to hold a workshop involving relevant experts on public engagement, consultation, and participation in public policy making. The purpose of this workshop is to inform members about the premises, principles, and purposes of public engagement activities and disseminate best practices.

The participants recommended that communications, public engagement, and dialogue should be incorporated into upcoming deliberations about changes to planetary protection policy:

Recommendation 9: COSPAR should encourage its members and the associated states to undertake public dialogue and engagement efforts at the national and/or regional level concerning ethics in space exploration, with the ultimate purpose of having public sentiment (including public perception) integrated appropriately into COSPAR policy deliberations. In addition, COSPAR should ask the PPP and PEX groups to hold a workshop on public engagement, consultation, and participation in policy-making to inform members about the premises, principles, and purposes of public engagement activities and best practices.

\section{Conclusion}

This Workshop discussed current planetary protection practices and policies and explored diverse perspectives on questions of whether planetary protection measures and practices should be extended to protect other aspects of planetary environments, going beyond "science protection" exclusively. Discussions resulted in an important and useful understanding of the intersections between science protection, protection of Earth, the utility of exploration and expansion into outer space, and the aesthetics and morality of humans relating to the physical (and perhaps biological) aspects of the natural universe. As an organization dedicated to scientific research, COSPAR has a historical role in the exploration and use of outer space to extend human knowledge, and has long espoused a policy that such research (especially when it might involve extraterrestrial life) should be done safely. It is only recently that COSPAR has envisioned its role in the use of outer space for other purposes and contemplated how far that role takes it into moral and aesthetic considerations.

Results of this Workshop were presented to COSPAR's Panel on Planetary Protection at the COSPAR Assembly in Bremen (2010). Subsequently, a number of events have taken place within COSPAR and without. In one area, it was determined that further discussion of changes to the COSPAR Planetary Protection Policy should take place at the 2012 COSPAR Assembly in Mysore, India. At Bremen, a further COSPAR workshop was approved on "Development of Foundational Ethical Principles Applicable to Planetary Protection and Space Exploration," which is to be held in cooperation with other organizations that have broader perspectives than is natural for COSPAR. Additionally, results of the Princeton workshop were reported at a COSPARled COPUOS symposium on "Planetary Protection and Space Exploration" held in Vienna in February 2011. Finally, in direct response to the public communications and awareness goals proposed by the Workshop and affirmed by the Panel on Planetary Protection in Bremen, the COSPAR Bureau took concrete steps to increase awareness of COSPAR's role in this critical area and cover an initial period of 6 years. To that end, COSPAR allocated $€ 51,500$ to support those activities. Elsewhere, international efforts to provide for the protection and use of outer space environments are gaining momentum. The future of planetary protection should be an interesting one, and its direction will be positively affected by the results that were deliberated as part of this Princeton meeting.

\section{Acknowledgments}

The Workshop organizers acknowledge the support of Dr. Catharine Conley (NASA Planetary Protection Officer), Dr. Gerhard Kminek (ESA Planetary Protection Officer) and Dr. Christopher Chyba (Princeton University, Woodrow Wilson School of Public and International Affairs) for their co-sponsorship of this workshop with COSPAR. Collectively the invited experts contributed in many ways to the deliberations and recommendations at the workshop.

The Princeton Workshop Participants included

(1) Jacques Arnould, CNES

(2) Amy Baker, Technical Administrative Services

(3) Linda Billings, George Washington University

(4) Penny Boston, New Mexico Institute of Mining and Technology

(5) Christopher Chyba, Princeton University

(6) Carol Cleland, University of Colorado

(7) Stephen Clifford, Lunar and Planetary Institute

(8) Cassie Conley, NASA HQ

(9) Pascale Ehrenfreund, George Washington University

(10) Emily Foote, UCLA

(11) Joanne Gabrynowicz, University of Mississippi School of Law

(12) Gerda Horneck, DLR

(13) Armel Kerrest, University of Western Brittany

(14) Gerhard Kminek, ESA/ESTEC

(15) Mark Lupisella, NASA Goddard Space Flight Center

(16) Christopher McKay, NASA Ames Research Center

(17) T.C. Onstott, Princeton University

(18) David Paige, UCLA

(19) Ted Peters, Graduate Theological Union, Berkeley

(20) Susanna Priest, University of Nevada, Las Vegas

(21) Margaret Race, SETI Institute

(22) Richard Randolph, Kansas City University of Medicine and Biosciences

(23) Petra Rettberg, DLR

(24) John Rummel, East Carolina University

(25) Brandon Stackhouse, Princeton University

(26) Elspeth Wilson, University of Pennsylvania

(27) Jean-Claude Worms, European Science Foundation

\section{Abbreviations}

COPUOS, Committee on the Peaceful Uses of Outer Space; COSPAR, The Committee on Space Research; IISL, International Institute for Space Law; NRC, National Research Council; PEX, Panel on Exploration; PPP, Planetary Protection Panel.

\section{References}

Cockell, C.S. and Horneck, G. (2004) A planetary park system for Mars. Space Policy 20:291-295. 
COSPAR. (2011) COSPAR Planetary Protection Policy (20 October 2002, amended 24 March 2011). COSPAR, Paris. Available online at http://cosparhq.cnes.fr/Scistr/PPPolicy\%20 (24Mar2011).pdf.

DeVincenzi, D.L., Stabekis, P.D., and Barengoltz, J.B. (1983) A proposed new policy for planetary protection. Adv Space Res 3:13-21.

Hargrove, E.C., editor. (1986) Beyond Spaceship Earth: Environmental Ethics and the Solar System, Sierra Club Books, San Francisco.

Lupisella, M. and Logsdon, J. (1997) Do we need a cosmocentric ethic? Paper IAA-97-IAA.9.2.09, presented at the International Astronautical Congress, Turin, American Institute of Aeronautics and Astronautics, Reston, VA.

McKay, C.P. (1990) Does Mars have rights? In Moral Expertise, edited by D. MacNiven, Routledge, London, pp 184-197.

McKay, C.P. (2009) Biologically reversible exploration. Science 323:718.

NRC. (2006) Preventing the Forward Contamination of Mars, The National Academies Press, Washington, DC.

Randolph, R., Race, M.S., and McKay, C.P. (1997) Reconsidering the theological and ethical implications of extraterrestrial life. The Center for Theology and the Natural Sciences 17:1-8.

Rolston, H., III. (1986) The preservation of natural value in the solar system. In Beyond Spaceship Earth: Environmental Ethics and the Solar System, edited by E.C. Hargrove, Sierra Club Books, San Francisco, pp 140-182.
Rummel, J.D., Race, M.S.A., and Horneck, G., editors. (2012) COSPAR Workshop on Ethical Considerations for Planetary Protection in Space Exploration. COSPAR, Paris. Available online at http://cosparhq.cnes.fr/Scistr/PPP Reports/PPP Workshop Report_Ethical Considerations.pdf.

United Nations. (1967) Treaty on Principles Governing the Activities of States in the Exploration and Use of Outer Space, Including the Moon and Other Celestial Bodies. Article IX, U.N. Doc. A/RES/2222/(XXI) 25 Jan 1967, TIAS No. 6347.

United Nations, Office for Outer Space Affairs. (2010) Space debris mitigation guidelines of COPUOS. United Nations, Vienna. Available online at http://www.unoosa.org/pdf/bst/ COPUOS_SPACE_DEBRIS_MITIGATION_GUIDELINES.pdf.

Address correspondence to: Dr. Margaret Race SETI Institute-CSC

189 Bernardo Ave Mountain View, CA 94043

E-mail: MRaceMom@aol.com

Submitted 20 June 2012 Accepted 18 July 2012 